\title{
SUBSTITUIÇÃO PARCIAL OU TOTAL DE ÓLEO DE CANOLA POR LECITINA DE SOJA EM RAÇÕES PARA LARVAS DE JUNDIÁ (Rhamdia quelen), PISCES, PIMELODIDAE $^{1}$
}

\section{PARTIAL OR TOTAL REPLACEMENT OF CANOLA OIL BY SOYBEAN LECITHIN IN DIETS FOR SOUTH AMERICAN CATFISH (Rhamdia quelen) LARVAE, PISCES, PIMELODIDAE}

\author{
Oclecio Uliana $^{2}$ José Henrique Souza da Silva $^{3}$ João Radünz Neto ${ }^{4}$
}

RESUMO

\begin{abstract}
O trabalho foi realizado no Setor de Piscicultura do Departamento de Zootecnia da Universidade Federal de Santa Maria, em um sistema para criação de larvas com recirculação de água termoregulada. O objetivo foi avaliar a substituição de óleo de canola por lecitina de soja na alimentação de larvas de jundiá durante os primeiros 21 dias de vida. No experimento, testaram-se sete tratamentos com três repetições, usando-se diferentes proporções de óleo de canola (0 a $5 \%$ ) e lecitina de soja $(0$ a $4 \%)$, incorporadas em uma ração preparada com fígado bovino e levedura de cana. Os melhores resultados foram obtidos no tratamento com $2 \%$ de lecitina de soja. Com ele, as larvas de jundiá apresentaram $80,25 \%$ de sobrevivência, peso médio individual de $184,89 \mathrm{mg}$ e $28,03 \mathrm{~mm}$ de comprimento total, diferindo significativamente dos demais tratamentos. De acordo com os resultados obtidos no experimento, conclui-se que a lecitina de soja em nível de $2 \%$ é mais eficiente que a lecitina usada em combinação com óleo de canola na nutrição de larvas de jundiá.
\end{abstract}

Palavras-chave: lipídios, lecitina de soja, larvas, Rhamdia quelen.

\section{SUMMARY}

This report was carried out at the Animal Sciences Department - Fish Culture Sector of the Federal University of Santa Maria, RS, Brazil, in a system for larvae raising with thermoregulation and recirculation of water, in order to assess the replacement of canola oil for soy lecithin in the feeding of south american catfish (Rhamdia quelen) larvae during their 21 days of life. In the experiment, seven treatments with three replications were tested by using different proportions of canola oil (0-5\%) and soybean lecithin (0-4\%), added to a diet prepared with beef liver and sugar canne yeast. The best results were obtained in treatment containing $2 \%$ of soy lecithin, in which catfish larvae presented $80.25 \%$ survival rate, $184.89 \mathrm{mg}$ average individual weight and $28.03 \mathrm{~mm}$ total length, what significantly differs from the other treatments. According to the results obtained in the experiment, it is concluded that soy lecithin (2\%) is more efficient as a lecithin - canola oil combination in south american catfish larvae nutrition.

Key words: lipids, soybean lecithin, larvae, Rhamdia quelen.

\section{INTRODUÇÃO}

A piscicultura no estado do Rio Grande do Sul encontra-se em franca expansão e, entre as espécies nativas, o jundiá (Rhamdia quelen) apresenta qualidades especiais de cultivo, pois é de fácil reprodução e rápido crescimento, adaptando-se ao consumo de alimentos artificiais. Além disso, o mercado atual mostra-se ascendente para a comercialização de sua carne e derivados. A produção de alevinos desta espécie ainda é reduzida, especialmente devido a baixa taxa de sobrevivência alcançada. Os primeiros dias, após a absorção das reservas vitelinas, são os mais críticos para a

\footnotetext{
${ }^{1}$ Parte da Dissertação de Mestrado do primeiro autor apresentada ao Curso do Pós-graduação em Zootecnia, Universide Federal de Santa Maria (UFSM)

${ }^{2}$ Engenheiro Agrônomo, Mestre em Zootecnia, Bolsista CNPq.

${ }^{3}$ Engenheiro Agrônomo, PhD, Professor Adjunto, Departamento de Zootecnia, UFSM

${ }^{4}$ Engenheiro Agrônomo, Doutor, Bolsista CNPq, Professor Adjunto, Departamento de Zootecnia, UFSM, 97105-900, Santa Maria, RS.

E-mail: radunz@ccr.ufsm.br. Autor para correspondência.
} 
sobrevivência das larvas de peixe, pois as exigências nutricionais das larvas da maioria das espécies cultivadas ainda não foram determinadas, o que dificulta a utilização de alimentos artificiais. Em geral, acredita-se que as dietas devem ter alta atração e palatabilidade e serem de fácil digestibilidade, de forma que as larvas ao se nutrirem desse alimento possam atingir bons índices de sobrevivência e de crescimento. KERDCHUEN \& LEGENDRE (1994) enfatizam que o uso de alimento artificial na nutrição de larvas se mostra adequado devido a sua produção em grande quantidade com economia e praticidade.

Além das proteínas, os lipídios são fundamentais para o desenvolvimento das larvas de peixes. LEGENDRE $\boldsymbol{e t}$ al. (1995) que usaram alimentos secos à base de levedura e fígado bovino e testaram diferentes fontes de lipídios, usando o nível de $7,5 \%$ de incorporação, em rações para o bagre Heterobranchus longifilis encontraram o melhor desempenho com o óleo de palma e o pior com óleo de figado de bacalhau. Já ULIANA (1997) encontrou boas taxas de sobrevivência quando utilizou $5 \%$ de óleo de canola ou de fígado de bacalhau na larvicultura do jundiá ( $\boldsymbol{R}$. quelen). Além dos ácidos graxos fornecidos pelas fontes de lipídios, o desenvolvimento larval exige também uma quantidade de fosfolipídios para intensa formação de novos componentes celulares, sobretudo no período inicial do desenvolvimento, e a biossíntese de fosfolipídios na fase larval pode não ser suficiente para suprir as próprias necessidades (KANAZAWA, 1985). A lecitina de soja pode ser usada como fonte de fosfolipídios na alimentação das larvas, e sua composição em ácidos graxos $(60 \mathrm{~g}$ por $100 \mathrm{~g}$ de produto) é de $59 \%$ de ácido linoléico e 7\% de ácido linolênico, segundo MEYERS (1990). A vantagem da lecitina da soja é que ela pode ser usada como ingrediente alimentar, servindo como estabilizador vitamínico, protegendo as vitaminas A e E contra oxidações, e também servindo como fonte de colina, inositol e outros componentes estimulantes do crescimento (MEYERS, 1990). O mesmo autor também sugere a taxa de 1 a $2 \%$ de lecitina de soja na dieta, variando a formulação da mesma de acordo com a espécie aquática cultivada, e em função do estádio de desenvolvimento. Assim, o presente trabalho foi realizado para avaliar o efeito da substituição parcial ou total de óleo de canola por lecitina de soja, visando melhorar os índices de produtividade na criação de larvas de jundiá.

\section{MATERIAL E MÉTODOS}

O presente trabalho foi realizado no mês de fevereiro de 1997, com duração de 21 dias, utilizando-se um sistema de criação de larvas com recirculação de água termoregulada, proposto por CHARLON \& BERGOT (1984). Constava de um sistema com 21 unidades experimentais, e um reservatório com capacidade de 1000 litros, dotado de um filtro biológico. Cada unidade experimental era constituída de duas bacias plásticas sendo, a interna com dimensão de 33 x 19 x $10 \mathrm{~cm}$, de formato retangular. Numa das extremidades havia uma abertura provida de tela de $300 \mu \mathrm{m}$ de abertura de malha que permitia a renovação de água. A bacia externa com dimensão de 34 x 23 x $11 \mathrm{~cm}$, de mesmo formato, possuía um dreno lateral que mantinha o nível da água da bacia interna. As larvas utilizadas no experimento foram contadas e distribuídas no sistema de criação, após absorção do saco vitelino, totalizando 160 larvas por unidade experimental.

A água do sistema de criação, originada de poço artesiano, foi mantida entre $24,0 \pm 2^{\circ} \mathrm{C}$ durante todo o experimento, utilizando-se vazões de $0,15 \ell / \mathrm{min}$ na primeira semana, $0,25 \ell / \mathrm{min}$ na segunda semana e $0,40 \ell / \mathrm{min}$ na terceira semana experimental, em cada unidade experimental. Os níveis de oxigênio permaneceram em torno de 7,5 \pm $1,0 \mathrm{mg} / \ell$ ao longo do experimento, e o pH foi mantido estável e neutro (próximo a 7,5). Os níveis de amônia e nitrito foram controlados e mantidos em níveis aceitáveis ao longo do experimento.

A composição dos dietas experimentais utilizadas encontra-se descrita na tabela 1. A composição centesimal das fontes proteicas usadas, expressa em matéria parcialmente seca é: levedura (Umidade: 6,35\%; $\mathrm{PB}=38 \%$; $\mathrm{EE}=0,35 \% ; \mathrm{FB}=$ $0,75 \% ; \mathrm{MM}=6,98 \%$ ) e do fígado bovino (Umidade: $7,03 \% ; \mathrm{PB}=66,38 \% ; \mathrm{EE}=12,94 \% ; \mathrm{FB}=0,0 \%$; $\mathrm{MM}=4,44 \%$ ). Os alimentos foram preparados no próprio setor de piscicultura da instituição, conforme metodologia descrita a seguir: - A levedura de cana foi previamente peneirada em malha de $75 \mu \mathrm{m}$, recuperando-se a fração granulométrica inferior para o preparo das rações. O fígado bovino cru também foi moído e misturado à levedura, vitaminas, lipídios

Tabela 1 - Composição das dietas experimentais (D), utilizadas na alimentação das larvas de jundiá (Rhamdia quelen), em percentagem.

\begin{tabular}{lrrrrrrr}
\hline INGREDIENTES & D1 & D2 & D3 & D4 & D5 & D6 & D7 \\
\hline Fígado bovino (MPS) & 29 & 34 & 34 & 34 & 34 & 30 & 34 \\
Levedura de cana & 60 & 50 & 50 & 50 & 50 & 57 & 50 \\
Premix vitamínico * & 10 & 10 & 10 & 10 & 10 & 10 & 10 \\
Premix mineral * & 1 & 1 & 1 & 1 & 1 & 1 & 1 \\
Óleo de canola & - & 1 & 2 & 3 & 4 & - & 5 \\
Lecitina de soja & - & 4 & 3 & 2 & 1 & 2 & -
\end{tabular}

* Segundo FONTINELLI (1997). 
e aos minerais, até completa homogeneização. Cada mistura foi então passada em máquina de moer carne formando "pelets", que foram secos ao sol a uma temperatura de $45 \pm 3^{\circ} \mathrm{C}$ durante 6 horas. Após esta etapa, as dietas foram novamente moídas e peneiradas separando-se as diferentes frações granulométricas, entre 100 a 200, 200 a 400 e 400 a $600 \mu \mathrm{m}$ que foram usadas na alimentação das larvas na primeira, segunda e terceira semanas experimentais, respectivamente. As rações foram acondicionadas em sacos plásticos e congeladas para melhor conservação até o seu uso.

A partir do dia seguinte da estocagem das larvas nas unidades experimentais, as mesmas foram alimentadas de meia em meia hora com uma pequena porção de ração, através de um alimentador automático controlado por um temporizador. Esta distribuição era feita ao longo de um período de 14 horas diárias, à vontade. A limpeza das bacias foi realizada diariamente, através de transferência das larvas para bacias limpas, sendo contabilizada a mortalidade.

Semanalmente, foi realizada biometria de uma amostra de 10 larvas por unidade experimental, previamente tranqüilizadas com tri-fenóxietanol na concentração de $0,15 \mathrm{ppm}$, medindo-se comprimento total e padrão (CT e CP) com o auxílio de lupa e papel milimetrado. No final do período experimental, as larvas sobreviventes em cada unidade experimental foram contadas, medidas e pesadas para obtenção do peso total. À partir desses resultados, foram determinadas a biomassa, a taxa de crescimento específico (TCE) e o Índice de sobrevivência real final.

O delineamento experimental foi inteiramente casualizado, com sete tratamentos e três repetições. Os dados obtidos no experimento foram submetidos a análise de variância e teste $\mathrm{F}$ ao nível de 5\% de significância, sendo aplicado o teste de Tukey para comparação entre as médias, usando-se o programa SAS (1995). Os dados de percentagem de sobrevivência foram analisados após transformação logarítmica de base 10 .

\section{RESULTADOS E DISCUSSÃO}

A dieta sem inclusão de lipídios resultou em 12,56\% de sobrevivência real aos 21 dias. As demais dietas contendo $5 \%$ de lipídio incorporado também apresentaram reduzida taxa de sobrevivência. As larvas alimentadas com a dieta D7 apresentaram a mais baixa taxa de sobrevivência, contrariando os dados obtidos por ULIANA (1997) quando usou uma dieta equivalente, com $5 \%$ de óleo de canola, em experimento realizado no mês de outubro/96, obtendo uma taxa de 95,57\%. A combinação de óleo de canola mais lecitina de soja não se mostrou eficiente, e somente o uso individual de lecitina na dieta D6 permitiu obter $80,25 \%$ de sobrevivência (Tabela 2). WIEGAND (1993) salienta que quando adicionado ao nível de $10 \%$ da dieta o óleo de canola poderia satisfazer os requerimentos de larvas de Carassius auratus em AGE (n-3 e n-6). Já POZERNICK \& WIEGAND (1997) utilizaram dietas com $43 \%$ de levedura e $43 \%$ de farinha de arroz comparando o óleo de canola com o de fígado de bacalhau (10\% da dieta) na criação de larvas e alevinos de Carassius auratus concluindo que a sobrevivência e o crescimento dos peixes foram comparáveis.

Estudos realizados por ALAMIDURANTE et al. (1991) com larvas de carpa comum (Cyprinus carpio), alimentadas com uma mistura de fígado e levedura (com proporções semelhantes às utilizadas neste trabalho), avaliaram essa dieta sem e com $5 \%$ de óleo de fígado de bacalhau, quando este último apresentou $64 \%$ de sobrevivência, $169 \mathrm{mg}$ de peso e $21 \mathrm{~mm}$ de comprimento total. Já o tratamento sem lipídio incorporado apresentou maior taxa de sobrevivência igual a $95 \%$, e larvas com 189mg de peso individual e $23 \mathrm{~mm}$ de comprimento total aos 21 dias; ou seja, a incorporação de $5 \%$ de óleo de fígado de bacalhau neste caso também acarretou um efeito negativo ao desenvolvimento das larvas de carpa. Fato semelhante ocorreu no presente trabalho, no qual o tratamento sem lipídio incorporado (D1) apresentou valores superiores nos parâmetros avaliados do tratamento D7 com 5\% de óleo de canola adicionado na dieta.

As larvas de jundiá, com o tamanho inicial (CT) igual a 5,5mm, eram menores que aquelas de experimento realizado durante o mês de

Tabela 2 - Peso médio individual (PMI), em mg, sobrevivência real (SR), em \%, biomassa, em mg, e taxa de crescimento específico (TCE) em \% / dia, das larvas de jundiá ao final do experimento.

\begin{tabular}{lcrrl}
\hline Tratamentos & PMI & SR & Biomassa & TCE \\
\hline D1 & $41,74^{\mathrm{c}}$ & $12,56^{\mathrm{b}}$ & $5,22^{\mathrm{b}}$ & $17,75^{\mathrm{c}}$ \\
D2 & $146,23^{\mathrm{a}}$ & $23,33^{\mathrm{b}}$ & $31,85^{\mathrm{b}}$ & $23,41^{\mathrm{a}}$ \\
D3 & $99,19^{\mathrm{abc}}$ & $18,71^{\mathrm{b}}$ & $19,98^{\mathrm{b}}$ & $21,85^{\mathrm{ab}}$ \\
D4 & $141,06^{\mathrm{ab}}$ & $21,02^{\mathrm{b}}$ & $26,53^{\mathrm{b}}$ & $23,49^{\mathrm{a}}$ \\
D5 & $95,51^{\mathrm{abc}}$ & $9,74^{\mathrm{b}}$ & $8,87^{\mathrm{b}}$ & $21,36^{\mathrm{abc}}$ \\
D6 & $184,89^{\mathrm{a}}$ & $80,25^{\mathrm{a}}$ & $149,40^{\mathrm{a}}$ & $24,85^{\mathrm{a}}$ \\
D7 & $52,14^{\mathrm{bc}}$ & $9,74^{\mathrm{b}}$ & $5,43^{\mathrm{b}}$ & $18,68^{\mathrm{bc}}$ \\
\hline
\end{tabular}

Médias seguidas por letras diferentes na coluna, apresentam diferenças significativas pelo teste de Tukey $(p<0,05)$. 
outubro/96 por ULIANA (1997) igual a 7,0mm. Assim, é provável que a lecitina de soja tenha sido mais importante na presente condição experimental em que as reservas endógenas das larvas poderiam ser mais escassas relacionada a uma pior condição corporal dos genitores. No experimento, já a partir da segunda semana, as larvas apresentaram diferenças significativas no comprimento, e as diferenças foram aumentando até os 21 dias. O tratamento D6 (2\% LS) foi o que apresentou melhor resultado com $28,03 \mathrm{~mm}$ de $\mathrm{CT}$, e $23,30 \mathrm{~mm}$ de $\mathrm{CP}$, diferindo significativamente dos demais tratamentos (Tabela 3). Por outro lado, larvas menores e mais fracas apresentaram melhor resposta com lecitina de soja, ao contrário do óleo de canola que, neste caso, apresentou as piores taxas de crescimento, observadas com o tratamento D7 $(15,38 \mathrm{~mm}$ de CT). $A$ adição de lecitina nos tratamentos D2 a D5 não melhorou o desempenho das larvas. GUERDEN $\boldsymbol{e t}$ al. (1995) constataram efeito positivo da inclusão de lecitina de ovo e verificaram que o aumento de 2 para $4 \%$ de PL nas rações experimentais não melhorou a sobrevivência ou crescimento durante os primeiros 14 dias de vida de larvas de carpa. Os autores salientam que para larvas de carpa o efeito de PL está relacionado com o requerimento para construção e renovação de membranas, que pode ser especialmente alto durante os estágios iniciais de rápido crescimento das larvas, que poderiam exceder a habilidade de síntese endógena de fosfolipídios. É enfatizado que uma especial atenção deve ser dada na suplementação adequada de PL quando as larvas são tratadas somente com rações, sem um alimento vivo rico em PL.

Também o peso médio individual (PMI) final do tratamento D6 se destacou atingindo $184,98 \mathrm{mg}$, levando a crer, assim como no caso do comprimento, nas larvas de jundiá com menor

Tabela 3 - Comprimento total (CT) e comprimento padrão (CP), em mm, das larvas de jundiá dos diferentes tratamentos (D).

\begin{tabular}{|c|c|c|c|c|c|c|c|c|}
\hline & \multicolumn{2}{|c|}{0 DIA } & \multicolumn{2}{|c|}{7 DIAS } & \multicolumn{2}{|c|}{14 DIAS } & \multicolumn{2}{|c|}{21 DIAS } \\
\hline & CT & $\mathrm{CP}$ & CT & $\mathrm{CP}$ & CT & $\mathrm{CP}$ & CT & $\mathrm{CP}$ \\
\hline D1 & 5,5 & 5 & $9,28^{\mathrm{b}}$ & $8,05^{\mathrm{c}}$ & $10,73^{b}$ & $9,35^{\mathrm{b}}$ & $16,77^{\mathrm{cd}}$ & $13,97^{b}$ \\
\hline D2 & 5,5 & 5 & $10,33^{\mathrm{ab}}$ & $8,98^{\mathrm{ab}}$ & $14,15^{\mathrm{ab}}$ & $12,13^{\mathrm{ab}}$ & $21,56^{b c}$ & $17,63^{b}$ \\
\hline D3 & 5,5 & 5 & $9,71^{\mathrm{ab}}$ & $8,55^{\mathrm{abc}}$ & $12,08^{b}$ & $10,33^{b}$ & $19,20^{b c d}$ & $15,83^{b}$ \\
\hline D4 & 5,5 & 5 & $10,28^{\mathrm{ab}}$ & $9,01^{\mathrm{ab}}$ & $12,63^{b}$ & $10,78^{b}$ & $22,56^{b}$ & $18,43^{b}$ \\
\hline D5 & 5,5 & 5 & $9,16^{b}$ & $8,30^{b c}$ & $10,70^{b}$ & $9,23^{b}$ & $18,27^{\mathrm{bcd}}$ & $15,06^{b}$ \\
\hline D6 & 5,5 & 5 & $10,85^{\mathrm{a}}$ & $9,41^{\mathrm{a}}$ & $17,26^{\mathrm{a}}$ & $14,50^{\mathrm{a}}$ & $28,03^{\mathrm{a}}$ & $23,30^{\mathrm{a}}$ \\
\hline D7 & 5,5 & 5 & $9,40^{b}$ & $8,28^{b c}$ & $10,60^{b}$ & $9,28^{b}$ & $15,38 \quad \mathrm{~d}$ & $12,83^{\circ}$ \\
\hline
\end{tabular}

Médias seguidas por letras diferentes na coluna, apresentam diferenças significativas pelo teste de Tukey $(\mathrm{p}<0,05)$. condição corporal o efeito individual da lecitina de soja é maior. Provavelmente, esse fato esteja relacionado com a presença de fosfolipídios na lecitina, principalmente fosfatidilcolina e fosfatidilinositol, componentes básicos da lecitina. Esses fosfolipídios são essenciais ao crescimento e sobrevivência, principalmente nos primeiros dias de alimentação, quando as larvas apresentam maior crescimento e aumento de peso, comparado em percentagem do seu peso inicial (MEYERS, 1990). Nos tratamentos com a mistura óleo de canola + lecitina não houve melhoria do PMI , verificando-se que a dieta com $5 \%$ de óleo de canola resultou em PMI extremamente baixo.

A biomassa foi influenciada pela baixa percentagem de sobrevivência na maioria dos tratamentos, já que, no final do experimento, as larvas sobreviventes eram as maiores em cada unidade experimental, pois as de menor tamanho não sobreviveram até o final do período (Tabela 2). No entanto, o tratamento D6 apresentou maior valor médio de biomassa (149,40mg) especialmente devido ao maior crescimento das larvas de jundiá e alta taxa de sobrevivência.

O maior valor de TCE foi constatado no tratamento D6 com 24,85\% / dia, diferindo significativamente dos demais tratamentos, e foi superior ao encontrado para larvas de bagre africano aos 14 dias experimentais, por KERDCHUEN \& LEGENDRE (1994), que utilizaram uma dieta composta por $50 \%$ de levedura, $35 \%$ de fígado bovino e com $5 \%$ de óleo de fígado de bacalhau. Como o crescimento dos peixes é muito alto durante o estádio de desenvolvimento de larvas e alevinos, pois os peixes podem aumentar cerca de $40 \%$ ou mais do seu peso/dia, no caso de larvas, ao passo que um peixe de $1 \mathrm{~kg}$ ganha em média menos de $1 \%$ por dia do seu peso (HEPHER, 1988), torna-se imperativo o uso de alimento que permita uma alta TCE. Assim sendo, a lecitina de soja usada isoladamente foi mais eficiente que o óleo de canola ou a mistura canola-lecitina na alimentação de larvas de jundiá. Nesse sentido novos trabalhos de pesquisa devem ser conduzidos para testar novas formas de apresentação da proteína de origem animal (fígado), com alternativas mais práticas de armazenagem e manuseio para elaboração de dietas para larvas de peixes.

\section{CONCLUSÃO}

Nas condições experimentais e na época do ano de realização deste trabalho, conclui-se que a lecitina de soja 
em nível de $2 \%$ é superior à lecitina usada em combinação com óleo de canola.

\section{AGRADECIMENTOS}

Agradecemos à Piscicultura ÁGUA DOCE pela doação das larvas utilizadas no presente trabalho de pesquisa e à PROVIMI S.A. pelos ingredientes usados nas rações experimentais.

\section{REFERÊNCIAS BIBLIOGRÁFICAS}

ALAMI-DURANTE, H., CHARLON, N., ESCAFFRE, A.M., $\boldsymbol{e}$ al. Suplementation of artificial diets for common carp. (Cyprinus carpio L.) larvae. Aquaculture, v.93, p.167-175, 1991.

CHARLON, N., BERGOT, P. Rearing system for feeding fish larvae on dry diets. Trial with carp (Cyprinus carpio) larvae. Aquaculture, v.41, p.1-9, 1984.

FONTINELLI, E. Efeitos do uso do concentrado protéico de soja, com e sem suplementação de aminoácidos, sobre o crescimento e sobrevivência da larvas de jundiá (Rhamdia quelen). Santa Maria, 1997. 38p. Dissertação (Mestrado em Zootecnia) - Curso de Pós-graduação em Zootecnia, Universidade Federal de Santa Maria, 1997.

GEURDEN, I., RADÜNZ NETO, J., BERGOT, P. Essentiality of dietary phospholipids for carp (Cyprinus carpio L.) larvae. Aquaculture, v.131, p.303-314, 1995.

HEPHER, B. Nutrition of pond fishes. Melbourne: Cambridge University, 1988. 388p.
KANAZAWA, A. Essential fatty acid and lipid requeriment of fish. In: COWEY, C.B., MACKIE, A.M., BELL, J.G. Nutrition and feeding in fish. London : Academic, 1985. p.281-298.

KERDCHUEN, N., LEGENDRE, M. Larval rearing of an African cattfish, Heterobranchus longifilis (Teleostei, Clariidae), a comparison between natural and artificial diet. Aquat Living Resour, v.7, p.247-253, 1994.

LEGENDRE, M., KERDCHUEN, N., CORRAZE, G., $\boldsymbol{e}$ t al. Larval rearing of on African catfish Heterobranchus longifilis (Teleostei, Clariidae): effect of dietary lipids on growth, survival and fatty acid composition of fry. Aquat Living Resour, v.8, p.363-365, 1995.

MEYERS, S.P. Aquaculture diets with lecithin. Feed International, Lousiana, june, p.17-19, 1990.

POZERNICK, M., WIEGAND, M.D. Use of canola oil in the feed of larval and juvenile goldfish, Carassius auratus (L). Aquaculture Res, v.28, p.75-83, 1997.

SAS. Statistical Analises System. User's Guide. Version 6.08, SAS INSTITUTE INC.4. ed. North Caroline : SAS INSTITUTE INC, 1995. 846p.

ULIANA, O. Influência de diferentes fontes e níveis de lipídios sobre a criação de larvas de jundiá (Rhamdia quelen), Pisces, Pimelodidae. Santa Maria, 1997. 66p. Dissertação (Mestrado em Zootecnia) - Curso de Pós-graduação em Zootecnia, Universidade Federal de Santa Maria. 1997.

WIEGAND, M.D. A study on the use of canola oil in the feed of larval goldfish, Carassius auratus L. Aquac Fish Management, v.24, p.223-228, 1993.

Ciência Rural, v. 31, n. 4, 2001. 